\title{
Three principles informing simulation-based continuing education to promote effective interprofessional collaboration: reorganizing, reframing and recontextualizing
}

\author{
Abstract \\ Introduction: Shoulder dystocia is a complex birth emergency where patient outcomes \\ remain a concern. This paper investigates the detailed processes of simulation-based \\ continuing education in a hospital where evidence over ten years demonstrates \\ improvements in practitioner knowledge, enacted practices, and maternal and child \\ outcomes. \\ Methods: Data were collected by video recording teams participating in a shoulder \\ dystocia simulation and debrief. Analysis combined grounded thematic development \\ with purposive coding of enactments of a relevant protocol (the ALSO HELPERR). \\ Results: Three themes were identified (three Rs) that capture how effective \\ interprofessional collaboration is promoted through collectively oriented reflection: \\ Reorganizing roles and responsibilities between team members; Reframing the problem \\ of shoulder dystocia from individuals correctly following a protocol, to a team of \\ professionals who need to attune to, respond to, and support one another; and \\ Recontextualizing by collectively 'commingling' theoretical knowledge with practical \\ experience to reflect on actions and judgements.
}

Discussion: The three Rs are relevant to diverse clinical settings, and address gaps in knowledge relating to the process of interprofessional simulation. Together they constitute a set of principles to inform the design and conduct of continuing education for interprofessional practice through simulation.

Keywords:

Simulation; interprofessional collaborative practice; obstetrics; emergency; shoulder dystocia 


\section{Background}

Safety and interprofessional collaboration are two linked aspects of quality that are a common focus of continuing education (CE). ${ }^{1,2}$ Research is especially needed to inform CE in care where risks to patients are high, and multi-professional teams need to collaborate in pressured environments. This is the case in shoulder dystocia - a complex birth emergency demanding a team of professionals to perform physically difficult actions amid serious risks to the mother and baby. $3,4,5$ Patient outcomes are a concern, often associated with maternal or foetal injury, and linked litigation against care providers. ${ }^{6}$ This paper explores simulation-based CE in a hospital where evidence over ten years demonstrates improvements in practitioner knowledge, enacted team practices, and maternal and child outcomes. ${ }^{7}$

The importance of conducting interprofessional education and training in teams is widely recognised. ${ }^{8,9,10,11}$ Single-profession approaches hamper the development of shared mental models, common language and clarity around other's roles. ${ }^{12}$ Educators can use the interprofessional demands of healthcare practice to create conditions for effective team training. ${ }^{10}$ Evidence is strong that simulation-based CE can promote effective interprofessional practice, especially if communication is explicitly addressed. ${ }^{1,2,13,14,15}$

However, there are gaps in understanding how best to conduct simulation to promote interprofessional practice. The interprofessional is often overlooked in debriefing, which has been found to favour individual actions and medical aspects. ${ }^{16,17}$ Researchers have also critiqued the limited strategies available to promote interprofessional practice, ${ }^{12}$ noting the challenges it presents to educators. ${ }^{10}$ These challenges include addressing relational dynamics, accommodating different professional identities and levels of experience in debriefing. ${ }^{8,10}$ Effective debriefing also needs to make strong connections between the simulation and clinical practice. ${ }^{2}$ Research is needed to elucidate the processes of interprofessional simulation and debriefing. ${ }^{10}$

This paper addresses these gaps in knowledge by focusing on what happens during simulation and debrief, and tracing how this can scaffold interprofessional practice. It does so in a clinical context - shoulder dystocia - where research is critically needed. The importance of interprofessional practice in dealing with shoulder dystocia is widely recognised. ${ }^{6,18,19}$ Protocols to standardise safe practice have been developed, and there have been widespread responses to calls for CE focusing on shoulder dystocia. ${ }^{6,20,21,22}$ Despite this, changes in practices and improvements in patient outcomes remain elusive in many settings. ${ }^{7,23,24}$ Better addressing the interprofessional aspects of shoulder dystocia is crucial. $3,23,25,26$ 
Thematic competency frameworks for interprofessional collaboration point at important aspects of this including clarification of roles and responsibilities, values and ethics, communication and team function are some important knowledge domains identified. ${ }^{27,28,29}$ While many studies have looked at short-term outcomes of educational programs, ${ }^{8,9,11,12}$ few have explored processes in CE contexts where longer term outcomes have been empirically demonstrated. This paper focuses on an interprofessional simulation CE program, delivered since 2008 in a Swedish hospital, called Practical Obstetric team-training (PROBE).

PROBE explicitly addresses interprofessional practices in diverse obstetric contexts, including shoulder dystocia. While practitioner knowledge and confidence, and the application of preferred management techniques are important indicators of successful training for shoulder dystocia, patient outcomes are regarded as the 'gold standard' measure. ${ }^{15,26}$ Evidence linked to PROBE spans all these domains, over a 10-year period, from pre-implementation (2004-2007) to $2015 .{ }^{7}$ Over this time, the number of diagnosed shoulder dystocias increased from $0.1 \%$ to $2.5 \%$ of births, but brachial plexus injuries reduced from $73 \%$ to $17 \%$, and fractures to foetus' clavicle and humerus also dropped. Documentation of actual practices in delivery improved from $63 \%$ to $93 \%$ of cases. A questionnaire found staff confidence in handling shoulder dystocia increased from $48 \%$ to $62 \% .{ }^{7}$ PROBE thus constitutes a valuable site at which to investigate simulation-based $\mathrm{CE}$. This paper offers an in-depth analysis of what happens in PROBE scenario and debriefs, focusing on how simulated action and debriefing support participants' learning to work together interprofessionally.

\section{Shoulder dystocia}

Shoulder dystocia, when a baby's shoulder jams against the mothers symphysis pubis after the head has birthed, constitutes a serious emergency. It can result in maternal or neonatal injury, paralysis or death. ${ }^{3}$ It is unpreventable and tends to occur without warning ${ }^{4}$ and is a major cause of anxiety in obstetric practice ${ }^{5}$ Associated injuries often lead to litigation and compensation claims.

Shoulder dystocia requires 'rapid and well-coordinated intervention by the health care team, some of whom may not have worked together before'. 3, p. 192 It is common enough to threaten patient safety, but not frequent enough that practitioners develop confidence in dealing with it through routine practice. ${ }^{20}$ As in many clinical contexts, protocols have been developed to guide and standardise practice. 
The ALSO HELPERR protocol was developed as part of the Advanced Life Support in Obstetrics course in the United States, and now often features in practice guidelines. ${ }^{6,21,30}$ It combines a memory aid (the mnemonic 'HELPERR') with a specific sequence of manoeuvres that avoid ineffective or dangerous practices (such as applying fundal pressure), increase the functional space in the pelvis, and rotate the baby to resolve the dystocia. ${ }^{6,21,23}$ Table 1 summarises the mnemonic and what each letter stands for. The manoeuvres are not unique to HELPERR - the protocol emphasises the sequence of them, including the anterior shoulder being addressed before the posterior one, because the latter is generally regarded as harder to perform.

[Table $1 \quad$ Summary of the ALSO HELPERR ${ }^{20}$ ]

There is often need to attempt the second $E$ and first $R$ several times, and again if the mother is rolled onto all fours (second R) ${ }^{20,21}$ Practitioners frequently have to switch who performs the internal manoeuvres, and guidelines often state the need to note elapsed time since the dystocia was noticed. ${ }^{31}$

PROBE uses the HELPERR protocol in training for shoulder dystocia. Analysis of the practices documented in the hospital where PROBE was implemented found increased practices aligning with HELPERR. ${ }^{7}$ In light of this evidence, the analysis presented here includes a focus on how PROBE participants actually enacted HELPERR, as well as on the debrief discussions that followed.

\section{Shoulder dystocia and continuing education}

Multiple reviews have found practitioners struggling to perform the manoeuvres and being unable to coordinate actions among the team. ${ }^{6,18,19}$ Regular CE for shoulder dystocia is therefore widely recommended, and simulation has been popular as an approach. ${ }^{20,22,23}$ Evidence from programs in the US and UK suggests simulation can: reduce harmful actions and increase safe actions, including as prescribed in HELPERR; ${ }^{32,33}$ improve knowledge in obstetric emergency management; ${ }^{34}$ reduce neonatal injury and improve patient outcomes. . $^{5,18,24,35}$

However, outcomes are not universally strong or positive. ${ }^{7,23,24}$ There is a growing view that to improve outcomes, CE needs to better address interprofessional and teamrelated aspects. . $^{4,23,25,26}$

Although training for shoulder dystocia typically focuses on the individual skills of the accoucheur [a person who assists during childbirth, often an obstetrician], 
effective multi-professional team working remains essential. Training individuals, rather than teams, may lead to the omission of critical steps. ${ }^{23,}$ p.3

\section{Methodology}

PROBE was introduced for staff at [name to be inserted] in 2008. It is run six times per year at the clinical training centre. The facilitators are midwives and obstetricians, and have completed an ALSO course in Obstetrics, as well as training in simulation debriefing provided within the hospital. All facilitators have been involved since its inception.

Obstetricians, midwives and nurse assistants have to attend PROBE once every 1.5 years. Each session lasts 3 hours, comprising two simulation scenarios and one practical skills training station. One of the scenarios always involves a shoulder dystocia. A debriefing follows each simulation scenario, following three stages, all of which fold patient outcomes and team collaboration together, rather than addressing them separately. ${ }^{2}$ The stages are:

1. Description: retelling what happened in the scenario, with the facilitator referring to notes made during the scenario;

2. Analysis: clarification of what the team had done well, and should continue to do;

3. Application: formulation of what has been learned and what to bring to clinical practice.

This staged approach is similar to Steinwachs ${ }^{\prime 36}$ model but has been adapted in PROBE to foreground interprofessional teamwork. Steinwachs ${ }^{36}$ model is based on the same broad approach (description, analysis, application), but the debrief in PROBE is more explicitly oriented towards working as a team.

Addressing gaps in knowledge about the processes of interprofessional simulation and debriefing requires data that captures how different professionals act and reflect in relation to interprofessional scenarios. To meet these needs, data were collected through video recording simulation scenario and debriefs. Three cameras provided multiple lines of sight. Given the shoulder dystocia itself is typically resolved within a few minutes, it was important to have video rather than hand-written observation notes, enabling the researchers to pause and re-play during analysis and consider the same issue from multiple standpoints. ${ }^{37}$ 


\section{Recruitment and Sampling}

In June 2018, all three PROBE shoulder dystocia scenarios and debriefs were video recorded at the Clinicum. One of the six days on which PROBE runs each year had been picked at random, and all clinicians completing the simulations that day were asked by a third party for their consent. None declined. Ten clinicians participated, one team of four and two teams of three. Each team comprised two midwives and an obstetrician; some also involved a nursing assistant.

The two facilitators also gave their informed consent. The lead facilitator was a senior consultant in obstetrics, and the second facilitator was a midwife (these roles are reversed in other scenarios). The former's role was to observe the simulation and clinical information (heart beats etc.), make notes, and take the lead in facilitating the debrief. The latter held the simulated foetus (ensuring there was a dystocia) and played the voice of the mother in the simulation; she supported her colleague in facilitating the debrief. No coaching or feedback was provided during the scenario, but clinical information that could not be observed in the available equipment was provided verbally.

Ethics approval limited video recording to three teams / one day in order to minimise disruption to the PROBE program. Findings were presented to other PROBE facilitators and they indicated that the recorded sessions were typical of how PROBE simulations and debriefs are conducted, and that the findings apply to the program more generally.

\section{Method}

The scenarios involved the use of a pelvic mannequin, a doll to simulate the foetus/baby, and a range of equipment that would be found in a delivery suite (drip, heart monitors, forceps, vacuum extractor, needles, foetal assessment machines etc.). On the day in question, participants completed two other sessions, one practical skills training focused on breech and twin deliveries, and one simulation session on postpartum haemorrhage. Participants were not told in advance that any of the sessions would involve a shoulder dystocia, nor given any reminders relating to HELPERR. Three shoulder dystocia simulation scenarios and debriefs were video recorded. Spoken interactions were transcribed verbatim and translated into English, with additional notes documenting relevant physical actions.

Data analysis 
The analytical process used purposive constant comparison methods ${ }^{38}$, iteratively combining a priori and grounded elements. ${ }^{39} \mathrm{~A}$ priori aspects included investigation of how HELPERR was enacted in scenarios and discussed in debriefs. Grounded elements involved the identification and verification of three emergent themes, presented below. These themes were specified in a codebook through a systematic approach to team-based qualitative analysis, using lay and technical definitions, and inclusion/exclusion criteria. ${ }^{40}$ The codebook was developed by three authors, and qualitatively validated by the fourth who sought clarification of definitions and application of the themes to the data, resulting in minor modifications to the thematic descriptors.

\section{Results}

The analysis found three key practices drove professionals' learning how to collaborate effectively in managing shoulder dystocia. Reorganizing roles and responsibilities between members of the birthing team; Reframing the problem of shoulder dystocia from performing correct actions to attuning to and supporting others while working together; and Recontextualizing by collectively 'commingling' theoretical knowledge with practical experience to reflect on actions and judgements.

Shoulder dystocia requires teams to redefine who does what, in what order, and with what tools arise. This was conceptualised through the analysis as reorganizing. PROBE provided opportunities to experience dynamic roles and responsibilities in practice, and to reflect on these through debriefing. In the simulation, as soon as the head retracted the teams reorganized their actions in line with HELPERR, shown in Table 2.

[Table $2 \quad$ Reorganizing practice when shoulder dystocia is noticed]

Once HELPERR was initiated, roles and responsibilities shifted as different team members took turns attempting the manoeuvres:

Midwife 1: l'll try to resolve the shoulder. Come on! Push! [to Obstetrician] Obstetrician: How is it working? Let me know when it's time to rotate Midwife 1: No, it's not loosening, turn it, see if I can get the arm. No. [to Midwife 2] Will you try?

Midwife 2: [to Obstetrician] Will you try?

Obstetrician: Should we roll her over? I will try once. 
Midwife 2: [Moves in] Let's try again then. Good it's coming, I have loosened the shoulder.

In moments like these, practice was reorganized as participants switched in and out of trying elements of HELPERR. They also changed their roles in terms of focusing on available clinical information (heart beats etc.), and interacting with the mother. Rather than remaining with stable roles associated with particular professions, the participants enacted fluid roles associated with the changing demands of the clinical emergency.

In the debrief, facilitators prompted participants to reflect on such reorganization as key to dealing with dystocia as a team:

Facilitator 1: You should give it more time [before switching roles]

Midwife 1: Yes, it was way too short. I asked Midwife 2, and then you asked the Obstetrician

Midwife 2: I was thinking, someone more experienced than me.

Facilitator 2: You can go round the whole team, it is terribly hard work. It takes time. You have to rotate, you take responsibility for one minute.

The facilitators helped participants reflect on why switching roles was important, and how all members of the team need to be ready to step in and take responsibility for the manoeuvres. This informed participants' commitments to future actions in practice in the third phase of debrief, reinforced by the facilitators making connections to patient safety:

Midwife 1: I will absolutely do manoeuvre 1 for longer. I was done way too quickly. I was so stressed.

Facilitator 1: Just giving each manoeuvre a little longer, not going for the back shoulder because that is where we get plexus injuries. So stay on the first one a bit longer.

The teams also discussed reorganization in terms of changing responsibility for taking the lead and making decisions. The facilitators highlighted participants' individual and collective contributions, which helped them reflect on lessons learned for future practice:

Midwife 2: You made correct decisions. It is so fucking insecure in a room when everybody is waiting for everyone else. It's terrible.

Facilitator 1: Watching you work, there was no doubt you shifted who took the lead, sometimes Midwife 1, sometimes Obstetrician. And there was 
no doubt who had the role. When that person says something, we work accordingly.

Obstetrician: It doesn't necessarily have to be the doctor taking the lead. We collaborate together. It's not about prestige. That is something I take with me [for future practice].

The second key finding concerned the way shoulder dystocia became a question not simply of implementing a protocol, but one of a collective, interprofessional accomplishment. This was conceptualised in the analysis as reframing ${ }^{41}$. Shoulder dystocia was reframed from a problem requiring 'me' to remember a sequence and then perform relevant actions, to a problem requiring interactions, anticipation, and commentary to enable the team to work together.

In the first phase of debrief, the facilitators prompted participants to recount a web of connected actions, such that they described the simulation scenario in a relational way. This reframing was done by linking one person's account to another's: 'Before you $[\mathrm{x}]$ got there, what did you [y] say? You [y] did something before she [x] did that'.

This provided a basis for further learning in the second, analytical, phase. Rather than reflecting on individual performances, the teams reflected on how they had worked together, prompted by facilitators asking questions such as "What did we do well?". In one team, participants focused on communication: "We all communicated well with each other". Often a facilitator stepped in to reframe an individual action in terms of its significance for the team.

Obstetrician: To be calm, take it easy. I think I did that well. You do get stressed, but realistically, you do have sometime to understand what we are dealing with.

Facilitator: Which contributed to calmness in the whole room. That gives you all the chance to stop and think where you are.

The analysis also found that PROBE helped participants commingle theoretical understanding and practical experience. This was conceptualised as recontextualization - the use of specialist knowledge in conjunction with professional experience to discern salient features of what is happening. ${ }^{42}$ In PROBE this was a collective process, in which the teams pooled knowledge and experience in their reflections. The commingling was done together with a view to understanding collaborative practice rather than individual actions or judgements. 
Practical experience of how shoulder dystocia can vary was commingled with knowledge of HELPERR, anatomy and patient safety. This produced new collective understandings of why particular actions were significant. For example, there was discussion of why those performing suprapubic pressure and internal manoeuvres needed to speak aloud what they were doing: "It is not always the case that you can see, when it's crowded, you do not see". Participants drew on past experiences of crowded dystocias to enrich understandings of verbalization as a part of interprofessional collaborative practice.

Recontextualization also supported participants' commitments to future actions. When asked what their key learning was for the future, the importance of noting elapsed time was raised by a nursing assistant: "You should watch the clock. I didn't realise the importance before". A facilitator offered further recontextualization by making further connections to other shoulder dystocias and to non-emergency births:

You can always do that. It can happen in an ordinary case as well. If the head is out and the contraction ends, I always glance at the clock. You can say if it's only one contraction, I can wait for the next one.

Thus the experience of simulation was commingled with knowledge of normal births to arrive at commitments to future practice. This recontextualization contributed to planned future actions that would promote patient safety in dystocia and other births.

\section{Discussion}

Reorganizing, reframing and recontextualizing in other settings

The three Rs emerged through the analysis as ways to capture how PROBE simulations unfolded, and how the challenges of interprofessional debriefing ${ }^{10}$ were met. The authors re-examined the literature for evidence that these practices are not confined to PROBE. To do this, eight studies in different clinical, educational and international settings were examined. Details are provided in Table 3, which shows that the three Rs are indeed relevant to diverse simulation-based CE contexts, even though they have not been explicitly foregrounded or conceptualised in this way. Together, the three Rs capture important features of CE aiming to promote interprofessional collaborative practice: the need to address fluid roles and responsibilities (reorganize), help professionals expand from an individual to a collective focus (reframe), and collectively commingle knowledge and experience (recontextualise). 
[Table $3 \quad$ Evidence of the three Rs from other published studies]

[Includes links to: ${ }^{1,13,16,17,43,44,45,46,47}$ ]

New ideas for interprofessional continuing education

These findings offer distinctive insights into how simulation can be used to strengthen $\mathrm{CE}$ for interprofessional collaborative practice. As Figure 1 shows they are not used in a linear sequence; each creates opportunities for the others.

[Insert Figure 1]

Each $\mathrm{R}$ has implications for the design and conduct of CE for interprofessional practice through simulation, however the greatest value arguably lies in their use in combination, as happens in PROBE. The fact that existing literature suggests each $R$ can be found in practices elsewhere (Table 3), and that PROBE is based on a well-recognised approach to debriefing ${ }^{36}$ suggest that the three Rs might be implemented without significant disruption. A key lesson from PROBE concerns how the three Rs connect to and support each other, as shown in Figure 1. Their intentional use together can help facilitators enrich the interprofessional aspects of debriefing.

The three Rs address a lack of attention to interprofessional matters that have been noted in many debriefing practices. ${ }^{16}$ These findings stem from fine-grained study of the process of simulation and debriefing involving interprofessional teams - as has been called for in the literature. ${ }^{10}$ The three Rs reveal how the shift from individual to teambased CE can be accomplished in simulation ${ }^{8}$, and offer insights that are much-needed given the challenges recognised in conducting debriefing with interprofessional groups. ${ }^{10}$ The three Rs can help professionals give qualitative accounts not only of their practice, but of their changing understanding of and commitments to working interprofessionally. ${ }^{10}$

Reorganizing points to the importance of constructing scenarios in a way that requires participants to enact the rapidly changing roles and responsibilities that agile interprofessional collaborative practice involves. ${ }^{12}$ This will help practitioners meet the demand of flexible roles required in collaborative teamwork. ${ }^{46}$ Once educators have designed scenarios that prompt reorganizing, it is helpful to explicitly highlight this in the debrief, so that participants can understand the importance of agile working and reflect 
on their own shifting roles and responsibilities. One way this can be achieved, as in PROBE, is to begin the debrief with a connective recounting of what happened, rather than a set of role-by-role descriptions. This focuses instead on unpicking the significance of changing ways of responding to and supporting one another, rather than evaluating actions within fixed roles. This extends previous findings that responsibility for action is a fertile focus in debriefs aiming to promote team- rather than individuallyoriented reflection. ${ }^{17}$

Reframing highlights the importance of CE enabling practitioners to approach complex patient care in a different way. Instead of focusing on their own practice, attention is directed to the challenge of working with others. Participants may need to be guided, as instincts may often be to focus on themselves and avoid what they feel might be criticism of others. This can be done in debriefing by highlighting how particular actions were consequential for others. Facilitators can encourage this by individuals to reflect on how their actions helped others, or asking the group to comment on the value of what a participant did from their perspective in a different role. Doing so importantly goes beyond communication to incorporate mutual understanding of one another's roles. ${ }^{10}$ The concept of reframing speaks to prior research, which has found that collective discussion on chains of action is useful in promoting simulation participants to understand their contribution in relation to others. ${ }^{17}$

Recontextualizing reminds us that simulation-based CE can be resourced by encouraging participants to make connections with other situations. However this needs to be carefully supported as a reflective process of collectively commingling theory and experience to address issues of collaboration. ${ }^{42}$ This can then strengthen professionals' capacity to envision future actions and make commitments in their own practice to improve care and enhance patient safety. Research in interprofessional simulation has found that supporting reflection on alternative practices (ie. different to those performed in the simulation) can strengthen connections between simulation and clinical practice. ${ }^{2}$ Recontextualisation can be accomplished by referring to participants' prior experience, as well as what they might do (differently) in future. While it may be common for participants to refer to 'real' practice during simulation debriefs, the recontextualizing that worked so effectively in PROBE had a particular quality: it addressed interprofessional work and connections between practitioners, rather than individual performances in themselves. Expressed differently, this can come down to a shift from 'What should I do in future?' to 'What should we do in future?' or 'What should I do in future as part of an interprofessional team?'. Facilitators can encourage insights from clinical practice from all participants, regardless of their professional role or clinical seniority, and thereby use recontextualisation as a means to address challenges in interprofessional debriefing of entrenched professional hierarchies and diverse clinical 
seniority. ${ }^{10}$ Recontexualizing foregrounds what happens in clinical practice, rather than specific individual's knowledge or status.

\section{Limitations}

This study is limited through its focus on one particular setting. The PROBE approach to debriefing, while informed by a commonly used model ${ }^{36}$, is also distinctive. Debriefing informed by the three Rs might vary from other approaches, for example, feedback would not necessarily follow the 'bandwidth' approach favoured in Human Factors informed practices, where feedback is limited to instances where participants deviate from desired performance. ${ }^{48}, 49$ PROBE facilitators often supported discussion of what the team did well, developing insights into the collaborative practices through which this was accomplished, and promoting understandings of how particular actions contribute to patient safety. In this way, feedback was as much oriented to securing repeated performance of effective practice as it was to correcting simulated actions for the future.

\section{Conclusion}

Examining PROBE has been useful to uncover how interprofessional teamwork can be promoted around the use of a standardised protocol in shoulder dystocia. While there is evidence the practices that were found are present elsewhere, further research would be needed to demonstrate that the three Rs function similarly as means to scaffold teamwork in other emergency situations, or in the use of other protocols.

PROBE exemplifies an innovative, change-oriented approach to CE, backed by robust evidence of quality improvement, professional learning and enhanced patient safety. The three Rs can inform the design and delivering of CE promoting effective interprofessional collaborative practice across diverse healthcare contexts. 


\section{Lessons for practice}

- The simulation enabled health professionals to learn to use a protocol collaboratively in order to effectively treat shoulder dystocia

- Interprofessional collaborative practice was highlighted through a specific approach to debriefing that focused on how individual actions contributed to effective, fluid work as a team

- Findings from analysis of video data suggest reorganizing, reframing and recontextualizing may be useful principles for simulation educators to adopt and adapt in debriefing aiming to enhance interprofessional collaborative practice.

\section{Legend for Figures and Tables}

Figure 1

Table 1

Table 2

Table 3
Three Rs that support interprofessional practice-based learning Summary of the ALSO HELPERR ${ }^{20}$

Reorganizing practice when shoulder dystocia is noticed

Evidence of the three Rs from other published studies 


\section{References}

${ }^{1}$ Freeth D, Ayida G, Berridge EJ, et al. Multidisciplinary obstetric simulated emergency scenarios (MOSES): Promoting patient safety in obstetrics with teamwork-focused interprofessional simulations. $J$ Contin Educ Health Prof. 2009;29(2):98-104.

${ }^{2}$ Andersen P, Coverdale S, Kelly M, Forster S. Interprofessional simulation: Developing teamwork using a two-tiered debriefing approach. Clin Sim Nurs. 2018;20:15-23.

${ }^{3}$ McArdle J, Sorensen A, Fowler Cl, Sommerness S, Burson K, Kahwati L. Strategies to improve management of shoulder dystocia under the AHRQ safety program for perinatal care. J Obstet Gynecol Neonat Nurs. 2018;47:191-201.

${ }^{4}$ Baxley EG, Gobbo RW. Shoulder dystocia. Am Fam Physician. 2004;69(7):1707-1714.

${ }^{5}$ Fahey JO, Mighty HE. Shoulder dystocia: using simulation to train providers and teams. J Perinat Neonat Nur. 2008;22(2):114-122.

${ }^{6}$ Jenkins L. Managing shoulder dystocia: understanding and applying RCOG guidance. Br J Midwifery. 2014;22(5):318-324.

${ }^{7}$ Dahlberg J, Nelson M, Dahlgren MA, Blomberg M. Ten years of simulation-based shoulder dystocia training - impact on obstetric outcome, clinical management, staff confidence, and the pedagogical practice - a time series study. BMC Pregnancy Childb. 2018;18(1):361.

8 Olde Bekkink M, Farrell SE, Takayesu JK. Interprofessional communication in the emergency department: residents' perceptions and implications for medical education. Int J Med Educ. 2018;9:262270

${ }^{9}$ Ericson A, Lofgren S, Bolinder G, Reeves S, Kitto S, Masiello I. Interprofessional education in a studentled emergency department: A realist evaluation. J Interprof Care. 2017;31(2):199-206

${ }^{10}$ Boet S, Bould MD, Layat Burn C, Reeves S. Twelve tips for a successful interprofessional team-based high-fidelity simulation education session. Med Teach. 2014;36(10):853-857

11 Truta TS, Boeriu CM, Copotoiu SM, et al. Improving nontechnical skills of an interprofessional emergency medical team through a one day crisis resource management training. Medicine (Baltimore). 2018;97(32):e11828

12 Eisenmann D, Stroben F, Gerken JD, Exadaktylos AK, Machner M, Hautz WE. Interprofessional Emergency Training Leads to Changes in the Workplace. West J Emerg Med. 2018;19(1):185-192.

${ }^{13}$ Eddy K, Jordan Z, Stephenson M. Health professionals' experience of teamwork education in acute hospital settings: a systematic review of qualitative literature. JBI Database System Rev Implement Rep. 2016;14(4):96-137.

${ }^{14}$ Kumar A, Wallace EM, East C, et al. Interprofessional simulation-based education for medical and midwifery students: A qualitative study. Clin Sim Nurs. 2017;13(5):217-227.

${ }^{15}$ Rosen MA, Hunt EA, Pronovost PJ, Federowicz MA, Weaver SJ. In situ simulation in continuing education for the health care professions: a systematic review. $J$ Contin Educ Health Prof.

2012;32(4):243-254. 
${ }^{16}$ Nyström S, Dahlberg J, Edelbring S, Hult H, Abrandt Dahlgren M. Debriefing practices in interprofessional simulation with students: A sociomaterial perspective. BMC Med Educ. 2016;16(148):18.

17 Nyström S, Dahlberg J, Edelbring S, Hult H, Abrandt Dahlgren M. Continuing professional development: pedagogical practices of interprofessional simulation in health care. Stud Cont Ed. 2017;39(3):303-319.

${ }^{18}$ Crofts JF, Bartlett C, Ellis D, Hunt L, Fox R, Draycott TJ. Training for shoulder dystocia: A trial of simulation using low-fidelity and high-fidelity mannequins. Obstet Gynecol. 2006;108(6):1477-1485.

19 Hope P, Breslin S, Lamont L, et al. Fatal shoulder dystocia: a review of 56 cases reported to the Confidential Enquiry into Stillbirths and Deaths in Infancy. Br J Obs Gynecol. 2005;105(12):1256-1261.

20 Gobbo RW, Warren J, Hinshaw K. Shoulder dystocia. In: Leeman L, Quinlan JD, Dresang LT, Gregory DS, eds. ALSO: Advanced Life Support in Obstetrics provider manual. 8th ed. Leawood, Kansas:

American Academy of Family Physicians; 2017:I: 1-20.

21 Gobbo RW, Baxley EG. Shoulder dystocia. In: Baxley EG, Deutchman M, Atwood L, Murhy N, Yu J, eds. ALSO: Advanced Life Support in Obstetrics Provider Course Syllabus. 4th ed. Leawood, Kansas: American Academy of Family Physicians; 2000:I: 1-13.

${ }^{22}$ RCOG. Shoulder dystocia: Green-top Guideline No. 42. London: Royal College of Obstetricians \& Gynaecologists; 2012.

${ }^{23}$ Cornthwaite K, Crofts JF, Draycott T, Siassakos D, Winter C. Training for obstetric emergencies: PROMPT and Shoulder Dystocia. London: The Health Foundation; 2015.

${ }^{24}$ Crofts JF, Winter C, Sowter MC. Practical simulation training for maternity care--where we are and where next. Br J Obstet Gynecol. 2011;118 Suppl 3:11-16.

${ }^{25}$ Monod C, Voekt CA, Gisin M, Gisin S, Hoesli IM. Optimization of competency in obstetrical emergencies: a role for simulation training. Arch Gynecol Obstet. 2014;298(4):733-738.

${ }^{26}$ Siassakos D, Crofts JF, Winter C, Draycott TJ. Multiprofessional 'fire-drill' training in the labour ward. Obstet Gynaecol. 2009;11:55-60.

27 Interprofessional Education Collaborative (IPEC). Core Competencies for Interprofessional Collaborative Practice: 2016 Update. Washington, DC: Interprofessional Education Collaborative; 2016.

28 Interprofessional Education Collaborative Expert Panel (IPEC). Core Competencies for Interprofessional Collaborative Practice: Report of an Expert Panel. Washington, DC: Interprofessional Education Collaborative; 2011.

${ }^{29}$ Rogers GD, Thistlethwaite JE, Anderson ES, Abrandt Dahlgren M, Grymonpre RE, Moran M, Samarasekera DD. International consensus statement on the assessment of interprofessional learning outcomes. Med Teach. 2017;39(4):347-359.

${ }^{30}$ Huntley M, Dickson Smith J. Management of shoulder dystocia using the HELPERR mnemonic. $\mathrm{Br} \mathrm{J}$ Midwifery. 2017;25(4):240-244.

${ }^{31}$ Chauhan SP, Gherman R, Hendrix NW, Bingham JM, Hayes E. Shoulder dystocia: comparison of the ACOG practice bulletin with another national guideline. Am J Perinatol. 2010;27(2):129-136. 
${ }^{32}$ Crofts JF, Fox R, Ellis D, Winter C, Hinshaw K, Draycott TJ. Observations from 450 shoulder dystocia simulations. Obstet Gynecol. 2008;112(4):906-912.

${ }^{33}$ Draycott TJ, Crofts JF, Ash JP, et al. Improving neonatal outcome through practical shoulder dystocia training. Obstet Gynecol. 2008;112(1):14-20.

${ }^{34}$ Crofts JF, Ellis D, Draycott TJ, Winter C, Hunt LP, Akande VA. Change in knowledge of midwives and obstetricians following obstetric emergency training: a randomised controlled trial of local hospital, simulation centre and teamwork training. Br J Obstet Gynecol. 2007;114(12):1534-1541.

35 Weiner C, Samuelson L, Collins L, Satterwhite C. 5-year experience with PROMPT (PRactial Obstetric Multidisciplinary Training) reveals sustained and progressive improvements in obstetric outcomes at a US hospital. Am J Obstet Gynecol. 2014;210(Supplement Issue 1):S40.

${ }^{36}$ Steinwachs B. How to facilitate a debriefing. Simul Gaming. 1992;23(2):186-195.

${ }^{37}$ Heath C, Hindmarsh J, Luff P. Video in Qualitative Research: Analysing Social Interactions in Everyday Life. Cambridge: Cambridge University Press; 2010.

${ }^{38}$ Boeije HA. A purposeful approach to the constant comparative method in the analysis of qualitative interviews. Qual Quant. 2002;36(4):391-409.

${ }^{39}$ Srivastava P, Hopwood N. A practical iterative framework for qualitative data analysis. Int J Qual Methods. 2009;8(1):76-84.

40 MacQueen KM, McLellan E, Kay K, Milstein B. Codebook development for team-based qualitative analysis. Field Methods. 1998;10(2):31-36.

${ }^{41}$ Hopwood N, Mäkitalo Å. Learning and expertise in support for parents of children at risk: A culturalhistorical analysis of partnership practices. Oxf Rev Educ. 2019.

42 Guile D. Professional knowledge and professional practice as continuous recontextualisation: a social practice perspective. In: Young M, Muller J, eds. Knowledge, expertise and the professions. Abingdon: Routledge; 2014:78-92.

${ }^{43}$ Owen JA, Brashers VL, Littlewood KE, Wright E, Childress RM, Thomas S. Designing and evaluating an effective theory-based continuing interprofessional education program to improve sepsis care by enhancing healthcare team collaboration. J Interprof Care. 2014;28(3):212-217.

${ }^{44}$ Yang LY, Yang YY, Huang CC, et al. Simulation-based inter-professional education to improve attitudes towards collaborative practice: a prospective comparative pilot study in a Chinese medical centre. BMJ Open. 2017;7(11):e015105.

${ }^{45}$ Lindh Falk A, Hopwood N, Abrandt Dahlgren M. Unfolding practices: a sociomaterial view of interprofessional collaboration in health care. Professions \& Professionalism. 2017;7(2).

${ }^{46}$ Eikeland Husebø S, Abrandt Dahlgren M, Edelbring S, et al. Reflecting on interprofessional simulation. In: Abrandt Dahlgren M, Rystedt H, Lelländer-Tsai L, Nyström S, eds. Interprofessional simulation in health care: Materiality, embodiment, interaction. Dordrecht: Springer; 2019:139-171.

${ }^{47}$ Zubairi MS, Lindsay S, Parker K, Kawamura A. Building and participating in a simulation: exploring a continuing education intervention designed to foster reflective practice among experienced clinicians. $J$ Contin Educ Health Prof. 2016;36(2):127-132. 
${ }^{48}$ Dow AW, Salas E, Mazmanian PE. Improving quality in systems of care: solving complicated challenges with simulation-based continuing professional development. $J$ Contin Educ Health Prof. 2012;32(4):230-235.

49 Seagull FJ. Human factors tools for improving simulation activities in continuing medical education. $J$ Contin Educ Health Prof. 2012;32(4):261-268. 